PROCEEDINGS OF THE

AMERICAN MATHEMATICAL SOCIETY

Volume 129 , Number 11, Pages 3161-3166

S 0002-9939(01)05961-5

Article electronically published on April 17, 2001

\title{
FINITE GROUPS EMBEDDABLE IN DIVISION RINGS
}

\author{
T. Y. LAM
}

(Communicated by Lance W. Small)

\begin{abstract}
In a tour de force in 1955, S. A. Amitsur classified all finite groups that are embeddable in division rings. In particular, he disproved a conjecture of Herstein which stated that odd-order emdeddable groups were cyclic. The smallest counterexample turned out to be a group of order 63 . In this note, we prove a non-embedding result for a class of metacyclic groups, and present an alternative approach to a part of Amitsur's results, with an eye to "demystifying" the order 63 counterexample.
\end{abstract}

\section{INTRODUCTION}

Finite groups that are embeddable in the multiplicative groups of division rings $K$ were completely determined by S. A. Amitsur $\mathrm{Am}$ in 1955. In case $K$ has characteristic $p>0$, the only possible finite subgroups of $K^{*}$ are cyclic groups, according to a theorem of I. N. Herstein $\mathrm{He}$. Thus, the only interesting case is when $K$ has characteristic 0 ; that is, when $K \supseteq \mathbb{Q}$.

In $[\mathrm{He}$, Herstein conjectured that odd-order subgroups of division rings $K$ were cyclic, and he proved this to be the case when $K$ is the division ring of the real quaternions $\mathbb{H}$. (This would have also followed from Coxeter's complete classification of finite subgroups of $\mathbb{H}^{*}$.) Herstein's conjecture for general division rings $K$ was settled negatively in $[\mathrm{Am}]$. As part of his complete classification of finite groups in division rings, Amitsur showed that the smallest noncyclic odd-order group that can be embedded in a division ring is one of order 63 (and this group is unique).

Amitsur's paper is daunting to read as it is long and technically complicated. In lecturing to a graduate class on division rings, I tried to find a simple reason for the "first exceptional odd order" 63 (to Herstein's conjecture). After some work, I did come up with a reason that was simple enough to be explained to my class, without having to go through any part of Amitsur's paper. Furthermore, the method I used led easily to the second exceptional odd order, 117 (which was not mentioned in Amitsur's paper). Since this line of reasoning did not seem to have appeared in the literature before, I record it in this short note. To better motivate the results discussed here, I have also included a quick exposition on the beginning part of the theory of finite subgroups of division rings. As a result, this paper can be read independently of $\mathrm{Am}$.

Received by the editors March 13, 2000.

2000 Mathematics Subject Classification. Primary 12E15, 16Kxx, 20B05; Secondary 20D20, 20B07, 16U60.

(C)2001 copyright retained by the author 


\section{BACKGround ON Amitsur Groups}

Let us say that a finite group $G$ is Amitsur if $G$ can be embedded in the multiplicative group $K^{*}$ of some division ring $K$. We start with some background information on such Amitsur groups. First the following easy observation.

(2.1) Theorem. Any abelian Amitsur group $G$ is cyclic.

Proof. Say $G \subseteq K^{*}$, where $K$ is a division ring. If $F$ is the center of $K$, then the $F$-span $L$ of $G$ is a finite-dimensional $F$-algebra. Since $L$ is a commutative domain, it must be a field. From field theory (see, e.g., [Ja, p. 132]), it then follows that $G \subseteq L^{*}$ is cyclic.

Next, we classify Amitsur $p$-groups. (Here, $p$ denotes any prime number, unrelated to the characteristic of our division rings.) This is possible thanks to some standard results in $p$-group theory.

(2.2) Theorem. Let $G$ be an Amitsur p-group. Then $G$ is cyclic if $p$ is odd, and $G$ is cyclic or dicyclic if $p=2$.

Recall that a group is dicyclic (of order $4 n$ ) if it is generated by two elements $x, y$ subject to the relations $x^{2 n}=1, y^{2}=x^{n}$, and $y x y^{-1}=x^{-1}$, where $n \geq 2$. Dicyclic groups are also known as generalized quaternion groups; they are 2-groups iff $n$ is a power of 2. It is easy to see that any dicyclic group $\langle x, y\rangle$ as above is embeddable in the division ring of real quaternions, so the conclusion in (2.2) is indeed the best possible.

Proof of (2.2). In the theory of $p$-groups, there is a well-known result which guarantees that, if a $p$-group has a unique subgroup of order $p$, then it satisfies the conclusion of the present theorem; see Th. 12.5.2 in $\mathrm{Ha}$. Thus, it suffices for us to verify that, if $G \neq\{1\}, G$ has a unique subgroup of order $p$. Say $G \subseteq K^{*}$, where $K$ is a division ring, with center $F$.

By elementary group theory, we know that $G$ has a nontrivial center. Fix a central element $x \in G$ of order $p$, so $|\langle x\rangle|=p$. If $\langle y\rangle \subseteq G$ is any subgroup of order $p$, then $L:=F(x, y)$ is a field. In this field, the equation $t^{p}-1=0$ has at most $p$ solutions, so we must have $\langle y\rangle=\langle x\rangle$, as desired.

In order to get some general results for non- $p$-groups, we introduce the following class of finite groups, where $m, n, r$ are natural numbers:

$$
G_{m, n, r}=\left\langle a, b: a^{m}=b^{n}=1, b a b^{-1}=a^{r}\right\rangle .
$$

Whenever we use this notation, it will be assumed that $r^{n} \equiv 1(\bmod m)$. This assumption guarantees that $G_{m, n, r}$ is a semidirect direct product of $\langle a\rangle$ and $\langle b\rangle$, with $|\langle a\rangle|=m$ and $|\langle b\rangle|=n$. In particular, $\left|G_{m, n, r}\right|=m n$. If necessary, we may assume $0<r<m$; but this is not always essential.

Let us say that a finite group $G$ is Sylow-cyclic if all of its Sylow subgroups are cyclic. 1 For instance, any $G$ with a square-free order is Sylow-cyclic, since each of its Sylow groups has a prime order. The following classical result of Hölder, Burnside, and Zassenhaus (see Th. 9.4.3 in [Ha]) gives a complete determination of all Sylow-cyclic finite groups.

\footnotetext{
${ }^{1}$ Such groups are also called $Z$-groups in the literature, after Hans Zassenhaus. We use the term "Sylow-cyclic" here since it is completely self-explanatory.
} 
(2.4) Hölder-Burnside-Zassenhaus Theorem. A finite group $G$ is Sylow-cyclic iff $G \cong G_{m, n, r}$ for some $m, n, r$ with $(m, n(r-1))=1$. (In particular, Sylowcyclic groups are solvable.)

Coupling this powerful classification result with (2.2), we deduce the following important information on a class of Amitsur groups, including all the ones of odd order.

(2.5) Theorem. If $G$ is an Amitsur group with order not divisible by 8, then $G$ is Sylow-cyclic, and hence $G \cong G_{m, n, r}$ for some $m, n, r$ with $(m, n(r-1))=1$.

According to this result, Amitsur groups with order not divisible by 8 are "fairly close" to being cyclic: they are at least metacyclic, i.e., extensions of cyclic groups by cyclic groups. To find all odd-order Amitsur groups, for instance, we must determine, for $m, n$ odd, which of the metacyclic groups $G_{m, n, r}$ are Amitsur (with $(m, n(r-1))=1)$. In the next section, we shall present some partial results in this direction, not only for odd-order groups, but for the groups $G_{m, n, r}$ in (2.3) in general. Note that the groups $G_{m, n, r}$ in this paper are "simpler versions" of the groups $G_{m, r}$ studied by Amitsur, in that the $G_{m, n, r}$ 's are given as semidirect products, while Amitsur's $G_{m, r}$ 's are not. As we shall see in the next section, the semidirect product representation of $G_{m, n, r}$ makes it possible for us to obtain quickly some necessary conditions for such a group to be Amitsur.

\section{NeCEssary CONDitions FOR The EMBEDDABILity of $G_{m, n, r}$}

In this section, we shall study the groups $G_{m . n, r}$ that are embeddable in division rings. (We do not need to assume the condition $(m, n(r-1))=1$.) The main result here is first stated in the following negative fashion.

(3.1) Theorem. Let $G=G_{m, n, r}$, where $n>1$ and $o(\bar{r})$ (the order of $\bar{r}$ in the unit group $\mathrm{U}\left(\mathbb{Z}_{m}\right)$ ) is exactly $n$. Then $G$ is not Amitsur.

Proof. Assume, instead, that $G \subseteq K^{*}$, where $K$ is a division ring. We use the presentation (2.3) for $G$. From $b a b^{-1}=a^{r}$, we get $b^{i} a b^{-i}=a^{r^{i}}$. Thus, $b^{i} a=a^{r^{i}} b^{i}$, and $a^{-1} b^{i} a=a^{r^{i}-1} b^{i}$. From $b^{n}=1$, we get $b^{n-1}+\cdots+b+1=0$ in $K$. Conjugating this equation by $a$, we get

$$
a^{r^{n-1}-1} b^{n-1}+a^{r^{n-2}-1} b^{n-2}+\cdots+a^{r-1} b+1=0 .
$$

Subtracting the last two equations and cancelling $b$ (from the right) gives

$$
\left(a^{r^{n-1}-1}-1\right) b^{n-2}+\left(a^{r^{n-2}-1}-1\right) b^{n-3}+\cdots+\left(a^{r-1}-1\right)=0 .
$$

Conjugating this by $a$ again and subtracting (3.3) from the resulting equation, we get after another cancellation of $b$ :

$$
\left(a^{r^{n-1}-1}-1\right)\left(a^{r^{n-2}-1}-1\right) b^{n-3}+\cdots+\left(a^{r^{2}-1}-1\right)\left(a^{r-1}-1\right)=0 .
$$

Carrying this process to the bitter end produces the equation

$$
\left(a^{r^{n-1}-1}-1\right)\left(a^{r^{n-2}-1}-1\right) \cdots\left(a^{r^{2}-1}-1\right)\left(a^{r-1}-1\right)=0 \in K .
$$

Therefore, $a^{r^{i}-1}=1$ for some $i \in[1, n-1]$. But then $r^{i} \equiv 1(\bmod m)$, which contradicts $o(\bar{r})=n$. 
In my lectures, I referred to the above as the "triple (-1)-proof", because of the three remarkable layers of $(-1)$ 's in the first factor of the key equation (3.4)! Note that this proof actually showed that, under the assumption that $o(\bar{r})=n>1$, $G_{m, n, r}$ cannot be multiplicatively embedded in any domain (that is, a nonzero ring without 0 -divisors). This, however, is not a real improvement, since it is easily seen that, once a finite (multiplicative) group $G$ is embeddable in a domain, then $G$ is in fact embeddable in a division ring.

We now record some consequences of (3.1).

(3.5) Corollary. If $G=G_{m, n, r}$ (generated by $a, b$ as in (2.3)) is Amitsur, then the largest square-free subgroup of $\langle b\rangle$ must be central in $G$. In particular, if $G=G_{m, n, r}$ is Amitsur and $n$ is square-free, then $G$ is cyclic.

Proof. For any prime $\ell$ dividing $n=o(b)$, let $\langle c\rangle$ be the $\ell$-Sylow group of $\langle b\rangle$, say of order $\ell^{t}$. Then $c a c^{-1}=a^{s}$ for some $s$, and $\langle a\rangle\langle c\rangle \cong G_{m, \ell^{t}, s}$ is still Amitsur. By (3.1), we must have $o(\bar{s})<\ell^{t}$ in $\mathrm{U}\left(\mathbb{Z}_{m}\right)$. This means that $c^{\ell^{t-1}}$ acts trivially on $a$ (by conjugation), and hence $c^{\ell^{t-1}}$ is central in $G$. From this, the first conclusion in the Corollary follows. If $n$ happens to be square-free, this conclusion means that $b$ is central in $G$. Thus, $G$ is abelian, and hence cyclic by (2.1).

(3.6) Corollary. Let $G$ be an Amitsur group of order $p^{t} q_{1} \cdots q_{k}$, where $k \geq 1$, and $p>q_{1}>\cdots>q_{k}$ are primes. Then $G$ is cyclic.

Proof. Since $k \geq 1, p$ is an odd prime. Thus, $G$ is Sylow-cyclic, and hence solvable by (2.4). We proceed by induction on $k$. Note that the solvability of $G$ implies, by Philip Hall's Theorem, that it has a subgroup $H$ of order $m:=p^{t} q_{1} \cdots q_{k-1}$ (see [Ha, Th. 9.3.1]). This Hall subgroup is still Amitsur, and hence cyclic by the inductive hypothesis. Also, since $[G: H]=q_{k}$ is the smallest prime divisor of $|G|$, $H$ must be normal in $G$. Thus, by taking a generator of $H$ and an element of order $q_{k}$ in $G$, we can represent $G$ as $G_{m, q_{k}, r}$ for some $r$. Since $q_{k}$ is a prime, (3.5) implies that $G$ is cyclic. Note that this argument is also sufficient to get the induction started, so the proof is complete.

A special case of (3.6) is that any square-free Amitsur group is cyclic. This recaptures Corollary 5 on p. 384 of [Am].

Note that, in (3.1) and (3.5), the integers $m, n$ and $r$ were arbitrary. To get some explicit numerical results, let us specialize these results to odd-order groups.

(3.7) Theorem. If $G$ is an Amitsur group of odd order $<171$, then $G$ is cyclic except possibly when $|G|=63$ or 117.

Proof. If $|G|$ is either a prime power, or square-free, or of the form $p^{t} q$ where $p>q$ are primes, the foregoing results imply that $G$ is cyclic. Among odd integers from 1 to 169 , the only ones not of any of the above forms are

$5 \cdot 3^{2}=45, \quad 7 \cdot 3^{2}=63, \quad 11 \cdot 3^{2}=99, \quad 13 \cdot 3^{2}=117, \quad 5 \cdot 3^{3}=135$ and $17 \cdot 3^{2}=153$.

If $|G|=45$ or $99, G$ is easily seen to be abelian (by Sylow theory), and hence cyclic (by (2.1)). Next, consider $|G|=135$. Since $G$ is Amitsur, we can represent it (thanks to (2.5)) in the form $G_{m, n, r}$ with $(m, n)=1$. We may assume $m, n>1$, so we have $m=5$ or $3^{3}$. But $\mathrm{U}\left(\mathbb{Z}_{5}\right)$ has no element of order 3 , and $\mathrm{U}\left(\mathbb{Z}_{3^{3}}\right)$ has no element of order 5 . Therefore, $G$ must be abelian and hence cyclic. The case $|G|=153$ can be treated similarly (with exactly the same conclusion). Thus, $G$ is cyclic in all cases, except possibly when $|G|=63$ or 117. 
What about $|G|=63$ ? There are, up to isomorphism, four groups of order 63; they are:

$$
\mathbb{Z}_{63}, \quad \mathbb{Z}_{3} \times \mathbb{Z}_{21}, \quad \mathbb{Z}_{3} \times G_{7,3,2}, \quad \text { and } \quad G_{7,9,2} .
$$

The first group is cyclic, and hence Amitsur. The second and third ones are not Sylow-cyclic, and hence not Amitsur. The remaining question is whether the noncyclic (but Sylow-cyclic) $G_{7,9,2}$ is Amitsur. Note that, since $o(\overline{2})=3<9$ in $\mathrm{U}\left(\mathbb{Z}_{7}\right),(3.1)$ does not apply to this group. Thus, there is a chance that $G_{7,9,2}$ is Amitsur. Indeed, the embeddability of this group was proved by Amitsur, and subsequent expositions on this have been given by C. Ford [Fo] and J. Dauns [Da]. For the sake of completeness, we give a quick sketch for the embeddability of $G_{7,9,2}$ below.

Let $L=\mathbb{Q}(\zeta)$ where $\zeta$ is a primitive 21 st root of unity, and let $\sigma \in \operatorname{Gal}(L / \mathbb{Q})$ be defined by $\sigma(\zeta)=\zeta^{16}$. Then $o(\sigma)=3$, so $L$ is a cubic extension of the fixed field $F:=L^{\sigma}$. Note that $F$ contains the primitive cubic root of unity $\omega:=\zeta^{7}$, since

$$
\sigma(\omega)=\zeta^{7 \cdot 16}=\zeta^{7}=\omega .
$$

On the other hand, for the primitive 7 th root of unity $a:=\zeta^{3}$, we have

$$
\sigma(a)=\zeta^{3 \cdot 16}=\zeta^{6}=a^{2} .
$$

Now introduce a new symbol $b$, and form the cyclic $F$-algebra

$$
K:=L \oplus L b \oplus L b^{2}, \quad \text { with } b^{3}=\omega, \quad \text { and } \quad b \ell=\sigma(\ell) b \quad(\forall \ell \in L),
$$

which has center $F$. We have $\operatorname{dim}_{\mathbb{Q}} F=\varphi(21) / 3=4$, so $\operatorname{dim}_{\mathbb{Q}} K=3^{2} \cdot 4=36$. In the algebra $K$, the elements $a, b$ satisfy the equations

$$
a^{7}=1, \quad b^{3}=\omega, \quad b^{9}=\omega^{3}=1, \quad \text { and } \quad b a=\sigma(a) b=a^{2} b .
$$

Therefore, $a$, and $b$ generate a group $G \cong G_{7,9,2}$ in the group of units $\mathrm{U}(K)$. It can be checked that $K$ is a division $F$-algebra (see, for instance, $[\mathrm{Fo}]$ ), so this establishes the noncyclic $G_{7,9,2}$ as an Amitsur group (of order 63). The center $F$ of the division ring $K$ is easily seen to be the biquadratic extension $\mathbb{Q}(\sqrt{-3}, \sqrt{-7})$ of the rationals. The group $G_{7,9,2}$ is rather well-known: Burnside described its complex irreducible representations as an exercise in [Bu, p. 319]. In this exercise, the reader was asked to show that the 3-dimensional complex irreducible representations of the group cannot be realized over their "character fields". By the general theory of Schur indices for group representations, this is in fact equivalent to the statement that the cyclic algebra $K$ constructed above is a division algebra. Thus, in a manner of speaking, Burnside already knew that $G_{7,9,2}$ was Amitsur, although, unfortunately, he did not give any clue as to how to prove his claim about its 3-dimensional complex irreducible representations.

The group $G_{7,9,2}$ has also found practical applications: recently, it has been utilized in the construction of efficient 3-antenna constellations, as reported by A. Shokrollahi in $\mathrm{Sh}$.

As for the case $|G|=117$, we have a noncyclic candidate $G_{13,9,9}$ (noting that $o(\overline{9})=3$ in $\left.\mathrm{U}\left(\mathbb{Z}_{13}\right)\right)$. This group can be shown to be Amitsur in the same way as $G_{7,9,2}$ was.

A substantial part of the argument in showing that a certain group is Amitsur is to establish that a certain $\mathbb{Q}$-algebra is a division algebra. This is by no means routine. In Amitsur's paper, this part of the work is handled by number-theoretic tools, 
such as the Hasse Norm Theorem and the Albert-Brauer-Hasse-Noether Theorem. Thus, to fully understand Amitsur's work would require considerable preparation in algebraic number theory.

In closing, we should mention that it is also of interest to fix a field $F$ (of characteristic zero), and to determine the finite groups $G$ that are embeddable in $K^{*}$ for some finite-dimensional central $F$-division algebra $K$. This problem has been studied by B. Fein and M. Schacher [FS], who called such $G$ " $F$-adequate groups". The embedding constructed for $G=G_{7,9,2}$ above showed that $G$ is $\mathbb{Q}(\sqrt{-3}, \sqrt{-7})$ adequate, but Fein and Schacher showed that $G$ is already $\mathbb{Q}(\sqrt{-3})$-adequate. This is remarkable, since Fein and Schacher have also proved the following interesting positive result on Herstein's conjecture: If $[F: \mathbb{Q}] \leq 2$, then any $F$-adequate odd-order group is cyclic, except when $F=\mathbb{Q}(\sqrt{-3})$ !

\section{REFERENCES}

[Am] S. A. Amitsur: Finite subgroups of division rings, Trans. Amer. Math. Soc. 80(1955), 361386. MR 17:577c

[Bu] W. Burnside: Theory of Groups of Finite Order, 2nd edition, Cambridge U. Press, 1911. (Reprinted by Dover Publications, 1955.) MR 16:1086c

[Da] J. Dauns: A Concrete Approach to Division Rings, Heldermann Verlag, Berlin, 1982. MR 84g:16014

[FS] B. Fein and M. Schacher: Embedding finite groups in rational division algebras, I, II, J. Algebra 17(1971), 412-428, and 19(1971), 131-139. MR 42:7702 MR 43:6261

[Fo] C. Ford: Finite groups and division algebras, L'Enseig. Math. 19(1973), 313-327. MR 49:7307

[Ha] M. Hall: The Theory of Groups, Macmillan Company, New York, 1959. MR 21:1996

[He] I. N. Herstein: Finite multiplicative subgroups of division rings, Pacific J. Math. 3(1953), 121-126. MR 14:1056j

[Ja] N. Jacobson: Basic Algebra, I, Second Edition, W. H. Freeman and Co., New York, 1985. MR 86d:00001

[Sh] A. Shokrollahi: Packing unitary matrices, Colloquium talk, University of California, Berkeley, Calif., November, 2000.

Department of Mathematics, University of California, Berkeley, California 94720

E-mail address: lam@math.berkeley.edu 